\title{
Structure Solution from Powder Data Using a Symmetry-Mode Parameter Set
}

\author{
B.J. Campbell, H.T. Stokes, G.L.W. Hart \\ Dept. of Physics \& Astronomy, Brigham Young University, Provo, Utah 84602, USA \\ J.W. Lewis, I.R. Evans, J.S.O. Evans \\ Dept. of Chemistry, Durham University, Durham, DH1 3LE, UK
}

Crystalline phase diagrams often contain multiple descendants of a common parent structure, each one possessing a distinct subgroup of the parent space-group symmetry. Relative to the parent, each child structure is distorted in some way, the details of which can be parameterized using symmetry modes of the parent space group rather than the traditional single-atom parameters (e.g. positions, occupancies, magnetic moments, and ADPs). Though the overall number of parameters is the same for the traditional and symmetry-mode descriptions, one can often isolate the principle features of the distorted structure using a relatively small number of symmetry modes, so that the effective complexity may be greatly reduced; the many inactive modes can be fixed at zero. Symmetry modes are basis functions of the irreducible representations of the parent symmetry group, and can be routinely calculated using programs such as ISODISTORT and directly against powder diffraction data using full-featured Rietveldanalysis packages. I'll present innovative Rietveld analyses from a variety of material families. 